\title{
Sonic Spray Nebulizer for Inductively Coupled Plasma Atomic Emission Spectrometry
}

\author{
Min Huang ${ }^{\dagger}$, Hisao KoJima, Atsumu Hirabayashi and Hideaki KoIzumi \\ Central Research Laboratory, Hitachi Ltd., Higashi-Koigakubo, Kokubunji, Tokyo 185-8601, Japan
}

\begin{abstract}
A novel nebulizer called a sonic spray nebulizer (SSN) was developed to introduce microliter sample solution for ICPAES with a high nebulization efficiency. At a sample uptake rate of $50 \mu 1 \mathrm{~min}^{-1}$, the detection limit of ICP-AES with the SSN is comparable to that with a conventional concentric nebulizer $(\mathrm{CCN})$ at a typical sample uptake rate of $850 \mu \mathrm{l} \mathrm{min}{ }^{-1}$. The absolute sensitivity of the SSN was improved by an average factor of 13 , compared to that of the CCN. The SSN system is capable of introducing a sample solution at a rate as low as $1 \mu 1 \mathrm{~min}^{-1}$ with a relative standard deviation (RSD) of about 2\%; therefore, the SSN nebulizer should be very useful for introducing biological or other samples in very limited volumes.
\end{abstract}

Keywords Nebulizer, microliter sample introduction, inductively coupled plasma atomic emission spectrometry

Inductively coupled plasma atomic emission spectrometry (ICP-AES) is widely used in various fields because of its high sensitivity and capability for simultaneous determining of most elements in the periodic table. ${ }^{1}$ Since the method was reported in the 1960's, research on sample introduction has been one of the most active areas. Many techniques have been developed to meet challenges from various kinds of samples and requirements. Although a solid sample can be introduced into the ICP by laser ablation ${ }^{2}$, arc discharge ${ }^{3}$, direct sample insertion ${ }^{4}$ or by preparing a slurry ${ }^{5,6}$, the predominant way is to introduce the sample solution with a conventional concentric nebulizer $(\mathrm{CCN})$.

Using the CCN nebulizer is simple, low cost, and convenient. However, its nebulization efficiency is quite low: $1-3 \%$. And the nebulizer requires a large sample volume, which is difficult in the cases of samples that are expensive, hazardous or limited in volume. Recently, direct injection nebulizer ${ }^{7,8}$, microconcentric nebulizer ${ }^{9}$, high efficiency nebulizer ${ }^{10-12}$ (HEN), oscillating capillary nebulizer ${ }^{13}(\mathrm{OCN})$ and direct injection high-efficiency nebulizer (DIHEN) ${ }^{14}$ were reported.

We aim to reduce sample uptake rate to the $\mu 1 \mathrm{~min}^{-1}$ level and to improve nebulization efficiency by using a microliter sample introduction technique. The technique would overcome the drawbacks of the $\mathrm{CCN}$ and reduce reagent consumption and waste emission at the same time. In this work, a sonic-spray nebulizer (SSN), based on sonic-spray ionization (SSI) technique ${ }^{15}$ which was originally used to ionize organic compounds in solution, was developed. The nebulizer was evaluated and compared with a conventional con-

$\dagger$ To whom correspondence should be addressed. centric nebulizer $(\mathrm{CCN})$.

\section{Experimental}

\section{SSN nebulizer}

In the $\mathrm{SSI}^{15}$, a silica capillary is fixed in the middle of an orifice formed in duralumin material. A sample solution is pumped through the silica capillary and dispersed into a fine aerosol by nitrogen gas passing through the orifice with high speed. Nitrogen gas at a flow rate as high as $31 \mathrm{~min}^{-1}$ is used to ionize organic analytes in the solution.

In this work, the SSN was constructed by improving SSI, as schematically shown in Fig. 1. The flow rate of the nebulizer gas was reduced to about $11 \mathrm{~min}^{-1}$ by reducing the orifice diameter from 400 to $250 \mu \mathrm{m}$. And the orifice was formed in polyamide material instead of duralumin in order to avoid contamination from the metal. A silica capillary (50- $\mu \mathrm{m}$ i.d. and $150-\mu \mathrm{m}$ o.d.) supported by a stainless-steel tube was put in the ori-

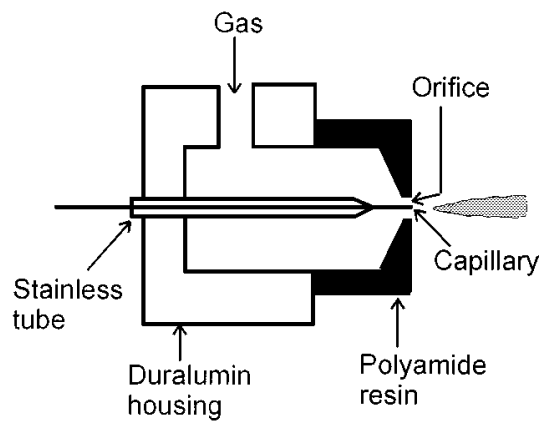

Fig. 1 Schematic diagram of the sonic spray nebulizer. 
fice. The stainless-steel tube was fixed through a ferrule. And the capillary was adjusted until its end was located right in the middle of the orifice to achieve good nebulization efficiency and stability. The sample solution was fed by a syringe pump (Micro-feeder, JPS1, Furue Science, Inc.) and a 1-ml syringe (Hamilton gastight syringe-1000, Hamilton Ltd.). Instead of using a metal-needle which may cause contamination, we use a homemade plastic connector joined by a Teflon tube. The gas pressure used was 5 atmospheric pressures. The flow rate of the nebulizer gas could be adjusted at the range of 0.2 to $1.81 \mathrm{~min}^{-1}$ by a thermal mass flow controller (Model 3650, Kofloc Ltd.), with an error of $2.0 \%$ for the flow scale used.

The SSN was directly inserted into a spray chamber endcap and sealed with two internal o-rings, which makes attaching or removing the SSN very convenient.

\section{Instrumentation and operating conditions}

The instrument used in this work was a combined simultaneous and sequential ICP atomic emission spectrometer (Jobin YVON, JY70PLUS). Operating conditions listed in Table 1 were used, unless otherwise specified.

\section{Reagents and chemicals}

Standard solutions of $\mathrm{As}, \mathrm{Au}, \mathrm{B}, \mathrm{Cu}, \mathrm{Ir}, \mathrm{Mg}, \mathrm{Pt}, \mathrm{Pd}$, $\mathrm{Se}, \mathrm{Sn}, \mathrm{Te}$ and $\mathrm{Zn}$ in $1 \% \mathrm{HNO}_{3}$ medium were prepared by diluting stock solution $\left(1000 \mu \mathrm{g} \mathrm{ml}^{-1}\right.$, SPEX Plasma Standard, SPEX industry, Edison, NJ, USA) with deionized water $(18 \mathrm{M} \Omega$ ) and high purity nitric acid to the concentrations required for determination. The blank solution was de-ionized water with $1 \% \mathrm{HNO}_{3}$.

\section{Results and Discussion}

\section{Optimization of flow rate of nebulizer gas}

In a CCN nebulizer, the sample uptake rate is mainly controlled by the nebulizer gas and this rate changed with the gas flow variation. In the SSN, however, the nebulizer gas and the sample uptake rate can be adjusted independently, without mutual limitation, because self-aspiration is significantly reduced in the SSN. This characteristic means that the nebulizer can be used for introducing a solution at low sample uptake rate.

Besides the nebulizer gas, an additional gas called a

Table 1 Instrumental conditions

RF generator: $40 \mathrm{MHz}$

Power: $1.0 \mathrm{~kW}$

Plasma gas: $121 \mathrm{~min}^{-1}$

Spray chamber: Scott type

Analytical observation height (mm): $15 \mathrm{~mm}$ above the coil

\begin{tabular}{lcc} 
Nebulizer: & CCN (Type C) & SSN \\
Nebulizer gas $\left(\mathrm{min}^{-1}\right)$ & 1.0 & 0.65 \\
Sample uptake rate $\left(\mu 1 \mathrm{~min}^{-1}\right)$ & 840 & 50 \\
\hline
\end{tabular}

coating gas can be added to the carrier gas before the aerosol enters the plasma torch. The coating gas was originally used to introduce fine droplets selectively into the plasma for a better precision and sensitivity in the CCN system. Nebulization with and without the coating gas was investigated in the SSN system. As shown in Fig. 2, when the total flow rate of carrier gas was kept the same in both cases, signal intensity obtained without the coating gas was higher than that obtained with $0.21 \mathrm{~min}^{-1}$ coating gas. Therefore, no coating gas was used in this work. And all of the carrier gas was used to disperse the sample solution. Even without the coating gas, the precision was better than that obtained with the CCN system. This result suggests that finer aerosol was produced with the SSN.

Although stable nebulization could be achieved at $0.31 \mathrm{~min}^{-1}$, a higher gas flow rate is necessary in order to improve nebulization efficiency. Figure 3 shows variation of signal intensity against the flow rate of nebulizer gas. For most elements, a maximum signal could be obtained at a flow rate of about $0.651 \mathrm{~min}^{-1}$. In the following experiments, the flow rate of the nebulizer gas was thus kept at $0.651 \mathrm{~min}^{-1}$.

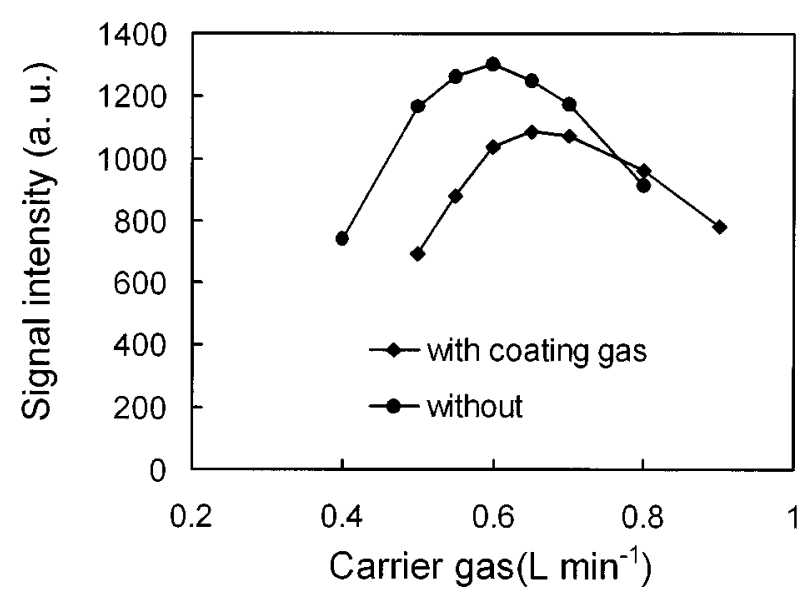

Fig. 2 Comparison of signal intensity obtained with and without a coating gas in the carrier gas.

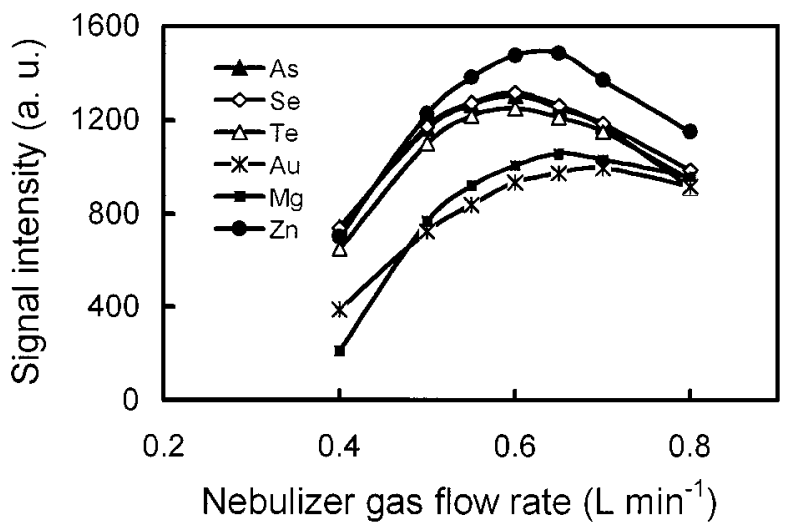

Fig. 3 Effect of nebulizer gas flow rate on signal intensity. 


\section{Introduction of microliter sample solution}

The thinness and small diameter of the capillary allow us to introduce and spray sample solution at the microliter level with good stability. It is not recommended to introduce the sample at more than $60 \mu \mathrm{l}$ $\mathrm{min}^{-1}$ because as the sample uptake rate is increased, the nebulization behavior shifts towards that of $\mathrm{CCN}$ and nebulization efficiency becomes poor. Our results show that the intensity increases linearly with the sample uptake rate when the rate is less than $40 \mu 1 \mathrm{~min}^{-1}$, but gradually reaches a maximum value, and then begins to decrease.

We want to introduce a sample solution at low sample uptake rate, although the sample uptake rate can be controlled from $1 \mu \mathrm{min}^{-1}$ to $120 \mu \mathrm{l} \mathrm{min}^{-1}$ in this system. At $1 \mu \mathrm{g} \mathrm{ml} \mathrm{m}^{-1}$, relative standard deviation (RSD) was found to be $2.0 \%$ for ten successive measurements of $5 \mu \mathrm{g} \mathrm{ml} \mathrm{m}^{-1} \mathrm{Cu}$ solution. This result, therefore, demonstrates the capability of introducing a sample solution at the microliter level in our system. This capability is very desirable for analysis of samples with a very limited volume. At $50 \mu \mathrm{min}^{-1}$, the RSD is about $1.5 \%$.

\section{Matrix effect}

The effect of salt content in a sample solution was investigated and calcium was selected as the matrix element. Figure 4 shows that there is no obvious signal depression when the concentration of $\mathrm{Ca}$ is less than $100 \mu \mathrm{g} \mathrm{ml}^{-1}$. Even at the matrix concentration of 500 $\mu \mathrm{g} \mathrm{ml^{-1 }}$, no clog of the nebulizer was observed. However, at this concentration, the signal intensities were depressed by $10.9 \%, 10.3 \%, 6.6 \%, 8.3 \%$ and $9.9 \%$ for Be, Cr, As, Se and La, respectively. This depression is mainly caused by variation of physical properties of the solution, such as liquid surface tension and coefficient of viscosity. Such phenomena can also be observed in other kinds of pneumatic nebulizers.

Figure 5 shows the long-term stability of the nebulizer. The variation of signal intensity was less than $3 \%$ when solution containing $200 \mu \mathrm{g} \mathrm{ml}^{-1}$ was continuously sprayed for $30 \mathrm{~min}$. Since the inner diameter of the

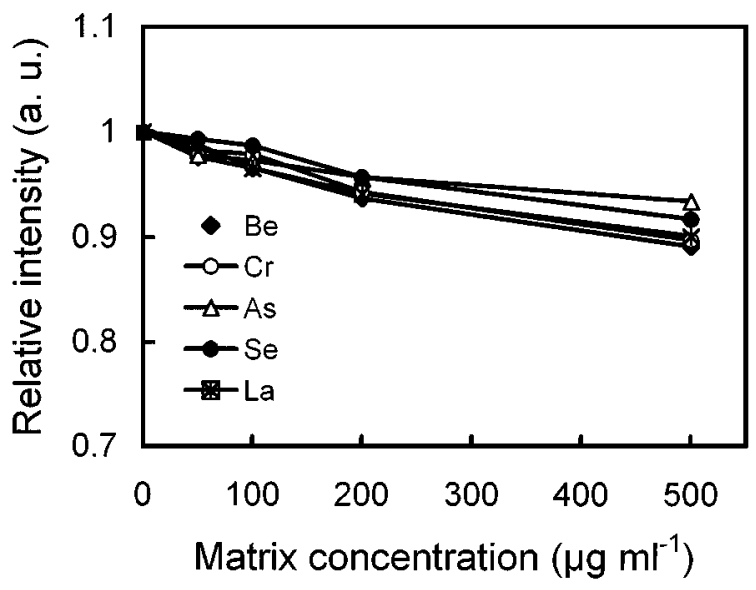

Fig. 4 Effect of Ca matrix on signal intensity. capillary in the SSN is much smaller than that of the $\mathrm{CCN}$, it is not recommended to introduce a sample solution with a salt content higher than $1000 \mu \mathrm{g} \mathrm{ml}^{-1}$ by the SSN.

\section{Detection limits}

The detection limit (DL) is defined as the concentration that produces a net intensity equivalent to three times the standard deviation of the background. DLs obtained with the SSN as well as those with the CCN are given in Table 2. At an uptake rate of $50 \mu \mathrm{min}^{-1}$, less than one tenth of that for the CCN, the DLs for the $\mathrm{SSN}$ are comparable to that for the $\mathrm{CCN}$. Considering the different sample uptake rates used in the two nebulizers, one can conclude that the absolute sensitivity of the SSN was improved by an average factor of 13 for the elements listed in Table 2, compared with the CCN. The ratio of DL for the SSN to that for the CCN varied

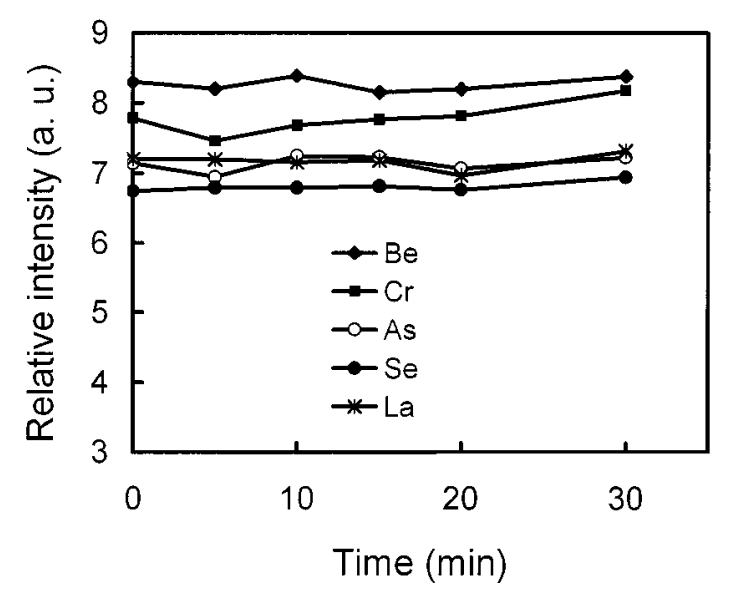

Fig. 5 Stability of nebulization of a sample solution containing a $200 \mu \mathrm{g} \mathrm{ml} \mathrm{g}^{-1} \mathrm{Ca}$ matrix.

Table 2 Comparison of detection limits of the SSN and the $\mathrm{CCN}$

\begin{tabular}{lllll}
\hline & \multirow{2}{*}{$\begin{array}{c}\text { Analytical } \\
\text { line/nm }\end{array}$} & \multicolumn{2}{c}{ Detection limit $^{\mathrm{a}} / \mathrm{ng} \mathrm{ml}^{-1}$} & \\
\cline { 3 - 4 } & & $\mathrm{CCN}$ & $\mathrm{SSN}$ & \\
\hline $\mathrm{As}$ & $193.759 \mathrm{I}$ & 0.057 & 0.070 & 1.2 \\
$\mathrm{Au}$ & $242.795 \mathrm{I}$ & 0.006 & 0.006 & 1.0 \\
$\mathrm{~B}$ & $249.773 \mathrm{I}$ & 0.005 & 0.007 & 1.5 \\
$\mathrm{Cu}$ & $324.75 \mathrm{I}$ & 0.0035 & 0.002 & 0.57 \\
$\mathrm{Ir}$ & $205.222 \mathrm{I}$ & 0.039 & 0.053 & 1.4 \\
$\mathrm{Mg}$ & $285.213 \mathrm{I}$ & 0.0014 & 0.0014 & 1.0 \\
$\mathrm{Pd}$ & $340.458 \mathrm{I}$ & 0.032 & 0.019 & 0.59 \\
$\mathrm{Pt}$ & $204.937 \mathrm{I}$ & 0.081 & 0.060 & 0.74 \\
$\mathrm{Se}$ & $196.090 \mathrm{I}$ & 0.028 & 0.081 & 2.9 \\
$\mathrm{Sn}$ & $253.484 \mathrm{I}$ & 0.057 & 0.047 & 0.82 \\
$\mathrm{Te}$ & $214.281 \mathrm{I}$ & 0.025 & 0.031 & 1.2 \\
$\mathrm{Zn}$ & $213.856 \mathrm{I}$ & 0.0015 & 0.002 & 1.3 \\
\hline
\end{tabular}

a. Sample uptake rates are $840 \mu 1 \mathrm{~min}^{-1}$ and $50 \mu 1 \mathrm{~min}^{-1}$ for the $\mathrm{CCN}$ and the SSN, respectively.

b. SSN/CCN indicates the ratio of the detection limit of SSN to that of $\mathrm{CCN}$. 
within a factor of 2, except the ratio of Se whose SD was found to be slightly worse.

Since the sensitivity improvement is almost independent of the elements, we attribute the improved sensitivity to the increase in nebulization efficiency. In the SSN, thinness of the capillary wall greatly improves nebulization behavior, compared with the thick wall configuration of the nozzle in the CCN. In addition, the capillary was axially fixed in the orifice in the SSN, which leads to better nebulization behavior. In a previous work, droplet sizes for most particles produced by the SSN were observed to be around $1 \mu \mathrm{m}^{16}$, which is much finer than that produced by the CCN. The mean diameter of droplets from a CCN was found to be about $5 \mu \mathrm{m} .{ }^{17}$

Finer droplet size of the aerosol not only increases amount of analyte introduced into the plasma, but also improves the analyte distribution inside the plasma. It is helpful in explaining the dynamic mechanism responsible for the improved sensitivity in the SSN to compare the fate of droplets produced by the two nebulizers.

Analytes in a droplet will experience vaporization, atomization and excitation processes after being introduced into the plasma. Generally, it takes a longer time for a bigger particle to be vaporized. With a $\mathrm{CCN}$, fine particles as well as considerable numbers of big particles are introduced into the plasma, the poor atomization efficiency for analytes in bigger particles and different atomization regions in ICP between the bigger and the smaller particles result in significant dilution of analyte. With the SSN, however, droplet size is small and relatively "homogenous", leading to a similar dynamic behavior inside the plasma for most of the droplets. Therefore, atoms of analyte produced in the plasma are concentrated in a narrower region; thus sensitivity is increased.

A part of this work (developing SSN) has been supported by CREST (Core Research for Evolutional Science and Technology) of the Japan Science and Technology Corporation (JST).

\section{References}

1. A. Montaser and D. W. Golightly (ed.), "Inductively Coupled Plasmas in Analytical Atomic Spectrometry", VCH Publishers, New York, Weinheim, Cambridge, 1992.

2. J. W. Carr and G. Horlick, Spectrochim. Acta, 37B, 1 (1982).

3. P. B. Farnsworth and G. M. Hieftje, Anal. Chem., 55, 1414 (1983).

4. E. D. Salin and G. Horlick, Anal. Chem., 51, 2284 (1979).

5. L. Ebdon and A. R. Collier, Spectrochim. Acta, 43B, 355 (1988).

6. M. Huang and X. Shen, Spectrochim. Acta, 44B, 957 (1989).

7. K. E. Lawrence, G. W. Rice and V. A. Fassel, Anal. Chem., 56, 289 (1984).

8. D. R. Wiederin, F. G. Smith and R. S. Houk, Anal.Chem., 63, 219 (1991).

9. F. Vanhaecke, M. Van Holderbeke, L. Moens and R. Dams, J. Anal. At. Spectrom., 11, 543 (1996).

10. H. Tan, B. A. Meinhard and J. E. Meinhard, Recent Investigations of Meinhard Concentric Nebulizers. Presented at the 19th Annual Meeting of the Federation of Analytical Chemistry and Spectroscopy Societies, Philadelphia, PA, September, 1992.

11. J. W. Olesik, J. A. Kinzer and B. Harkleroad, Anal. Chem., 66, 2022 (1994).

12. H. Liu and A. Montaser, Anal. Chem., 66, 3233 (1994).

13. R. F. Browner, Presented at the 21st Annual Meeting of the Federation of Analytical Chemistry and Spectroscopy Societies, St. Louis, MO, October 1995; Paper 8.

14. J. A. McLean, H. Zhang and A. Montaser, Anal. Chem., 70, 1012 (1998).

15. A. Hirabayashi, M. Sakairi and H. Koizumi, Anal. Chem., 66, 4557 (1994)

16. A. Hirabayashi and Juan Fernandez de la Mora, Intern. J. Mass Spectrom. Ion Process, 175, 277 (1998).

17. R. H. Clifford, I. Ishii and A. Montaser, Anal. Chem., 62 , 390 (1990).

(Received October 7, 1998)

(Accepted December 28, 1998) 